\title{
Anti-wettability of Chemically and Physically Modified Glass Surfaces
}

\author{
Suhad Sbeih ${ }^{1,2, *,(D)}$, Werner Steffen², Michael Kappl ${ }^{2}$ \\ ${ }^{1}$ School of Basic Sciences and Humanities, German Jordanian University, Amman 11180, Jordan \\ ${ }^{2}$ Max-Planck Institute for Polymer Research, Mainz, 55128 Mainz, Germany
}

*Correspondence author: E-mail: suhad.sbeih@gju.edu.jo; Tel.: +962 64294444 Ext. 4255

DOI: $10.5185 / \mathrm{amp} .2021 .010421$

The demand for new advanced functional materials has driven scientific work over the past decades. Nature has been inspiring in the creation of different types of self-cleaning and super repellent surfaces mimicking those of plants (lotus leaves), animals (shark skin) or insects (butterfly wings, water strider). To produce and maintain super repellent materials, chemical modification of the surface by using low surface energy materials such as fluoropolymers and/or siloxanes is necessary. Also, physical modification of surface roughness enhances super-repellency against various liquids. The surface roughness can be achieved e.g., by the deposition of nano particles (NPs) using Liquid Flame Spray (LFS). Industrial applications like paper coatings, oil-water separation, and microfluidic devices have benefited from the fabrication of superhydrophobic surfaces by LFS. In our work, glass substrates were fluorinated by chemical vapor deposition (CVD) method, and others were additionally pre-coated with silica NPs by LFS. The coated glass surfaces were characterized for their anti-wettability by measuring the contact angles of water and compare that to bare glass. The influence of the produced coatings on the wettability of surface with different liquids was examined through measuring advancing/receding contact angles as well as the roll off angle. Results showed that compared to bare glass only fluorination of glass increased the water static contact angle from $18^{\circ}$ to almost $112^{\circ}$. This is indicative of hydrophobic behaviour. Coating glass with silica NPs by LFS before fluorination, enhanced the water anti-wetting property for super hydrophobicity. LFS coating provided good oleophobic characteristic.

\section{Introduction}

In the past decade, much attention has been devoted to the fabrication of surfaces with potential for many applications involving liquids [1-3]. Anti-wetting materials have established themselves among the most frequently used substrates for applications involving self-cleaning, liquids separation and transportation, anti -biofouling, microfluidics devices, packaging, filters, optical switching, medical disposables, and electronics printing [4,5]. To make surfaces that nicely repel certain types of liquids, one needs to investigate the factors controlling the surfaceliquid interface such as the surface structure at a microscale. Nature always inspires scientists for linking the underlying details of materials composition and structures with their behavior. Lotus leaves are good example for superhydrophobic surface with a super water repellent property (for an overview of different plants with this ability see ref. 6). For many of these superhydrophobic surfaces they are originating from multiscale roughness [6-8].

Introduction of roughness to surfaces is achieved by different methods. Multiscale structures at nano- and micro- lengths that result in a porous coating and thus an entrapped air layer upon wetting are common strategy.
Nanoparticles (NPs), micro-filaments, pillar arrays, nanorods or flake-like structures can be deposited on surfaces by various procedures such as dip coating, spin coating, spraying, and polymerizations techniques, and particle growth. Also, chemical modification of the surface by using low surface energy materials such as siloxanes or fluoropolymers is necessary to achieve and maintain super repellent chemistry. The chemistry of the surface is adjusted by plasma deposition, chemical vapor deposition (CVD), or immersion methods. The structure modification and chemical treatment can be performed separately (by two-steps) or at once [9-11].

In the liquid flame spray (LFS) method, NPs are thermally synthesized in the flame and deposited on the substrate instantly resulting in multiscale roughness that originates from individual particles and their aggregates. This makes LFS one of the most promising approaches towards super repellent surfaces. The challenge is to ensure the high repellency not only against liquids with high surface tension (water, $72.8 \mathrm{mN} / \mathrm{m}$ ) but also lower surface tension (hexadecane, $27.5 \mathrm{mN} / \mathrm{m}$ ), as well.

A deposited water droplet on a solid smooth ideal surface, in a static equilibrium state, makes contact between three phases (solid-liquid-vapor), as shown Fig. 1. 




Fig. 1. Contact angle as defined by Young's equation.

The static contact angle $(\theta, \mathrm{CA})$ is defined by Young's equation as:

$$
\cos \theta=\frac{\gamma_{S V}-\gamma_{S L}}{\gamma_{L V}}
$$

where $\gamma_{S V} \quad \gamma_{S L} \gamma_{L V}$ are the interfacial tensions between solid-vapor, solid-liquid, and liquid-vapor $[\mathbf{1 2 , 1 3}]$.

Adding roughness to the surface can result in super hydrophobicity identified by a high static $\mathrm{CA}>150^{\circ}[\mathbf{8 , 9}]$. However, water adhesion to the surface is mainly affected by the wetting state of the surface. It can be more like a complete wetting (Wenzel state) [14] or a partial wetting (Cassie-Baxter state). The Wenzel state occurs when liquid is completely filling the space between the protrusions on the surface. The Cassie-Baxter state [15] defines a partial wetting where vapor pockets are trapped between the liquid and the solid. These two wetting models are wellestablished in literature and are based on the physical nature and chemical composition of the surface.

Experimentally, the wetting state can be inferred from the contact angle hysteresis $(\mathrm{CAH})$ and the sliding angle (SA). CAH is the difference between the advancing and receding contact angles that are measured when the threephase contact line of a droplet is forced to advance or retract, respectively. SA is the surface tilting angle at which the droplet starts sliding (rolling off). As a result, superhydrophobic surfaces with low $\mathrm{CAH}$ and SA are considered to have the self-cleaning effect (Lotus effect) while those with high $\mathrm{CAH}$ and SA are not $[\mathbf{8 , 1 0}$.

In this work, we investigate the anti-wettability behavior of water and hexadecane (as a low surface energy liquid) on glass substrates. The role of chemically modified glass surfaces was tested by measuring the CA values for a fluorinated glass surface and compare it to the bare glass. Also, the enhancement of the surface by roughness was tested by employing a home built LFS machine for the fabrication of silica NPs microlayer on glass, then characterize its anti-wettability through CA measurements.

\section{Experimental}

Microscope glass slides obtained from Thermo scientific (Menzel-Gläser, Germany) were used as our substrates. Chemical modification was achieved by pre-cleaning the substrate by ultrasonication with Acetone then with Ethanol solutions. Then the substrate was ultrasonicated and rinsed with DI water and blow dried by nitrogen. After that, a treatment by plasma cleaner (Diener Electronic Femto, $6 \mathrm{~cm}^{3} /$ min oxygen flow rate) for 10 minutes at $100 \mathrm{Watt}$. Germany) with oxygen gas was utilized for removing organic contaminants from surface and activate it with hydroxyl groups. This is followed by the CVD for surface activation with fluorine group. Samples were placed in a desiccator together with $1 \mathrm{~mL}$ of poly trichloro $1 \mathrm{H}, 1 \mathrm{H}, 2 \mathrm{H}-$ perfluorooctyl silane (PTFOS) 97\% Sigma Aldrich, at 100 mbar for 30 minutes.

For silica NPs coating by LFS, a flame was created by oxygen $(2 \mathrm{~L} / \mathrm{min})$ and methane $(1 \mathrm{~L} / \mathrm{min})$. A ready mixture of TEOS (98\%, Sigma Aldrich) as precursor for the particles dissolved in isopropanol (Fischer Scientific) was dispersed into the flame using oxygen (5 $\mathrm{L} / \mathrm{min}$ ). The concentration of TEOS in isopropanol was $370 \mathrm{mg} / \mathrm{mL}$.

For CA characterization, OCA35 goniometer (DataPhysics Instruments GmbH, Filderstadt, Germany) was utilized for performing the static CA, advancing and receding $\mathrm{CA}$, and sliding angles with water and hexadecane.

\section{Results and discussion}

Static contact angles were measured by the sessile drop mode method. A $4 \mu \mathrm{L}$ droplet was deposited on the to be tested surface at the rate of $0.5 \mu \mathrm{L} / \mathrm{s}$, then the droplet frame is fitted to find the left and right contact angles. The measured mean static CA of $18^{\circ}$ for water with bare glass was very low (Fig. 2a), indicative of a hydrophilic surface. Anti-wettability enhanced with the fluorinating of glass as the static CA measures $112^{\circ}$ (Fig. 2b). Adding roughness to the surface by LFS coating of silica NPs prior to the fluorination resulted in a super hydrophobic surface of a static CA of $153^{\circ}$ (Fig. 2c).
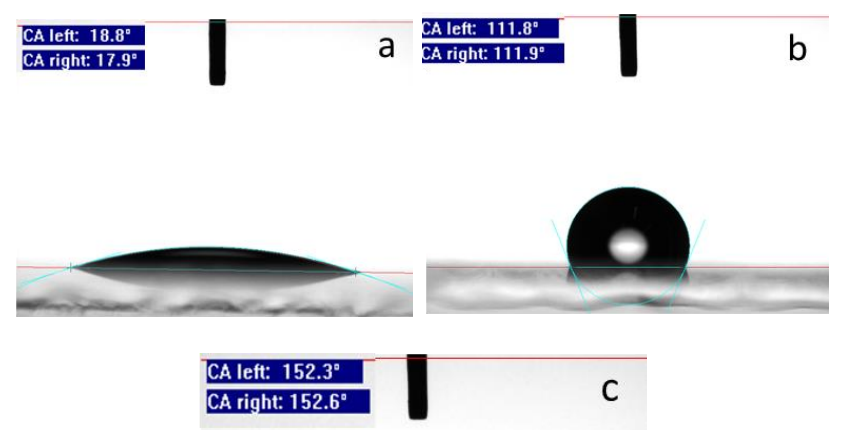
(b) only fluorinated surface, (c) silica NPs coating with fluorination. 


\section{Advanced Materials \\ Proceedings \\ www.vbripress.com/amp}

Advancing and receding contact angles ARCA were measured by the filling and extracting method through the sessile drop needle in mode. A $4 \mu \mathrm{L}$ droplet was deposited on the tested surface at the rate of $0.5 \mu \mathrm{L} / \mathrm{s}$, then retracted at the same rate. Two ARCA iterations were performed for each measurement. The ARCA measurements were performed for the only fluorinated and the silica NPsfluorinated surfaces with water (Fig. 3a and Fig. 3b, respectively), as they showed hydrophobic behavior seen through the static CA measurements. The mean angle is plotted as a function of the run number. Advancing and receding angles of $117^{\circ}$ and $93^{\circ}$, respectively, for the only fluorinated surface include the previously measured static $\mathrm{CA}\left(112^{\circ}\right)$ and give a CAH of $24^{\circ}$. That is indicative of a good hydrophobic behavior. Advancing and receding angles of $154^{\circ}$ and $145^{\circ}$, respectively, for the silica NPsfluorinated surface include the previously measured static $\mathrm{CA}\left(153^{\circ}\right)$ and give a $\mathrm{CAH}$ of $9^{\circ}$. That is indicative of a superhydrophobic behavior.
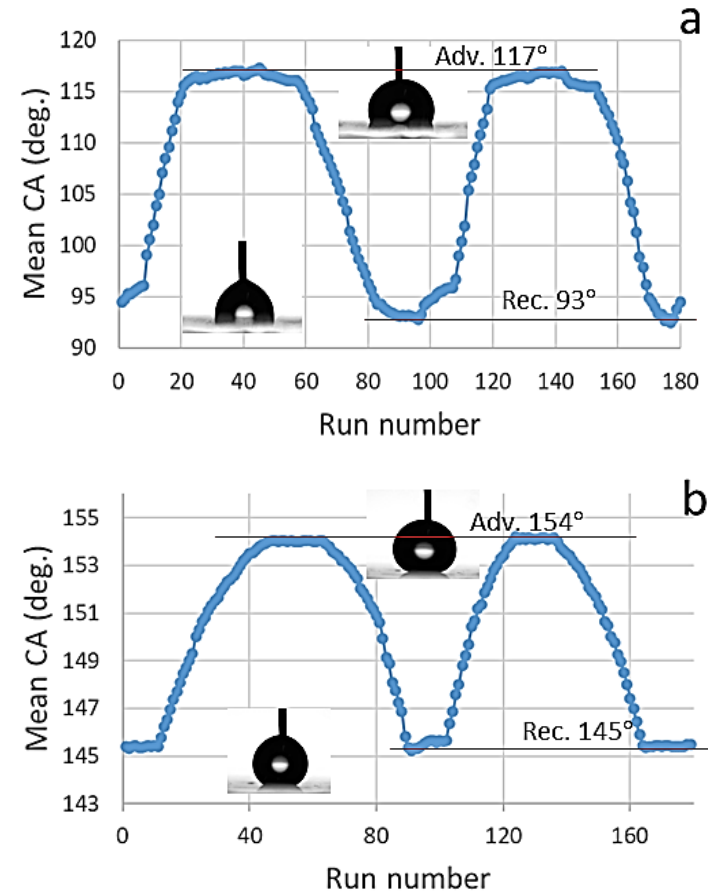

Fig. 3. ARCA results for the: (a) only fluorinated sample, and (b) silica NPs with fluorination sample.

Sliding angle (SA) angle measurements were performed for the two samples by depositing a $4 \mu \mathrm{L}$ droplet and then tilting the base at an angle rate of $0.67 \%$ s over the range $0-40^{\circ}$. The water droplet didn't slide over the only fluorinated surface; thus, it owns $\mathrm{SA}>40^{\circ}$.

Fig. 4 shows the mean $\mathrm{CA}$ as a function of the base tilt angle for water droplet on the silica NPs coated and fluorinated surface. The droplet rolled off the surface at the point when lost contact with surface and a sudden failure of imaging/fitting the droplet occurred, that is at a tilting angle of $7.5^{\circ}$. Such a low SA infers a superhydrophobic surface.

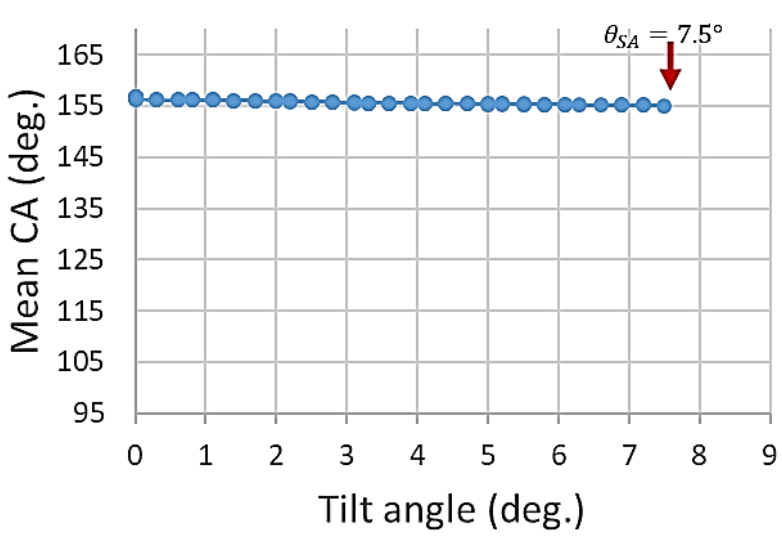

Fig. 4. Water SA test result for the silica NPs with fluorinated surface.

Hexadecane is a low surface energy liquid that spread easily on surfaces. CA measurements for hexadecane were used to probe the oleophobic characteristic of the studied surfaces. The hexadecane static CA measurements for the bare glass, only fluorinated, and silica NPs coatedfluorinated samples are shown in Figs. 5a, 5b, and 5c, respectively. The mean static $\mathrm{CA}$ for the bare glass was only $15.6^{\circ}$ indicating an oleophilic behavior. The only fluorinated sample attained a mean static CA of $75.5^{\circ}$ which is also relatively low and doesn't show an oleophobic behavior. However, the silica NPs coated and fluorinated sample read a mean static CA of $145^{\circ}$ which infers good oleophobic behavior.

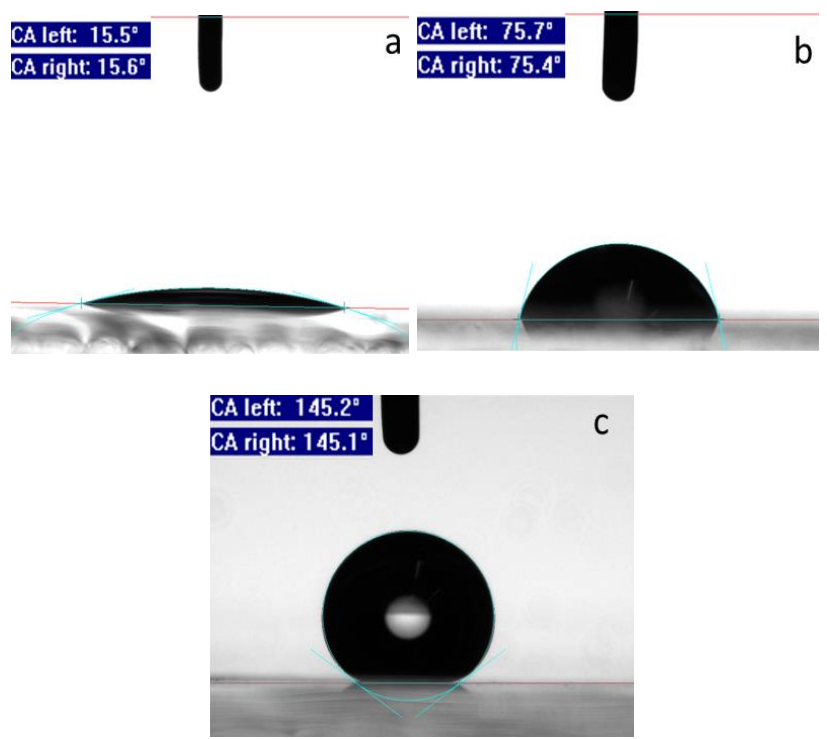

Fig. 5. Static contact angles with hexadecane for the surfaces: (a) bare glass, (b) only fluorinated surface, (c) silica NPs coating with fluorination.

For investigating the oleophobic behavior of the silica NPs coated and fluorinated surface, the hexadecane ARCA and SA angles for this surface were measured. Fig. 6a shows hexadecane ARCA result. Advancing and receding angles of $153^{\circ}$ and $132^{\circ}$, respectively, include the 


\section{Advanced Materials \\ Proceedings \\ www.vbripress.com/amp}

previously measured static CA for hexadecane with the surface $\left(145^{\circ}\right)$ and give a CAH of $21^{\circ}$. That is indicative of a good oleophobic behavior. Fig. 6b shows the mean CA as a function of the base tilt angle for hexadecane droplet on the silica NPs coated and fluorinated surface. The droplet slid the surface at the point when lost contact with surface at a tilting angle of $17.5^{\circ}$.


Fig. 6. Hexadecane a- ARCA and b- SA angle results for the silica NPs coated and fluorinated sample.

\section{Summary}

In this study we performed an investigation of the chemical and physical modifications effect on the anti-wetting property. Bare, only fluorinated, and silica NPs coating and fluorination on glass substrates were tested for their hydrophobic and oleophobic properties by conducting the CA measurements with water and hexadecane droplets. Results show that the chemical modification (fluorination) alone resulted in hydrophobic behavior. Adding roughness to the surface by LFS coating of silica NPs in addition to the fluorination, led to a superhydrophobicity with contact angles of more than $150^{\circ}$ and sliding angle of less than $10^{\circ}$. LFS coating provided a significant increment of static CA for hexadecane, with much lower surface tension, and reasonable values of $\mathrm{CAH}$ and $\mathrm{SA}$ angles indicating oleophobic behavior. LFS coatings might be further optimized towards super-olephobic surfaces by tuning LFS process parameters and silanization procedure.

\section{Future work}

LFS coatings of surfaces revealed promising macroscopic wetting characteristics against different liquids. The microscopic structure of these coatings can be manipulated by controlling the LFS process and parameters. Further investigation of these coatings on a microscopic scale is necessary to be able to optimize their macroscopic function and antiwetting behavior. Also, inspecting their durability and lifetime is of a high importance to be employed in possible practical applications.

Keywords: Fluorination, nanoparticles, liquid flame spray, self-cleaning, oleophobic.

\section{References}

1. Hu, D.; Yu, Q.; Yang, Y.; Weng, L.; Nanotechnology, 2020, 31, 175701.

2. Zhang, J.; Seeger, S. ; ChemPhysChem, 2013, 14, 1646-1651.

3. Yao, W.; Li, L.; Li, O.; Cho, Y.; Jeong, M.; Cho, Y.; Chemical Engineering Journal, 2018, 352, 173-181.

4. Liu, J.; Ye, L.; Sun, Y.; Hu, M.; Chen, F.; Wegner, S.; Mailänder, V.; Steffen, W.; Kappl, M.; Butt, H.; Advanced Materials, 2020, 1908008.

5. Han, J. T.; Xu, X.; Cho, K.; Langmuir, 2005, 21, 6662-6665

6. Neinhuis, C.; Barthlott, W.; Annuals of Botany, 1997, 79, 667-677.

7. Quéré, D.; Annual Review of Materials Research, 2008, 38, 71-99.

8. Teisala, H.; Tuominen, M.; Haapanen, J.; Aromaa, M. Stepien, M.; Mäkelä, J.; Saarinen, J.; Toivakka, M.; Kuusipalo, J.; Nordic Pulp and Paper Research Journal, 2014, 29, 4, 747-759.

9. Paven, M.; Fuchs, R.; Yakabe, T.; Vollmer, D.; Kappl, M.; Itakura, A.; Butt, H.; adv. Fun. Mater., 2016, 26, 4914-4922.

10. Teisala, H.; Tuominen, M.; Aromaa, M.; Makela, L. M.; Stepien, L.; Saarinen, J.; Toivakka, M.; Kuushipalo, J.; Surface \& Coatings Technology, 2010, 205, 436.

11. Wong, W. S.; Tricoli, A.; Advanced Coating Materials, 2018, 16, 393.

12. Butt, H.; Graf, K.; Kappl, M.; Physics and Chemistry of Interfaces, John Wiley \& Sons, 2013.

13. Young, T.; Philosophical Transactions of the Royal Society of London, 1805, 95, 65.

14. Wenzel, R. N.; Industrial and Engineering Chemistry, 1936, 28, 988 .

15. Cassie, A. B. D.; Baxter, S.; Nature, 1945, 155, 21.

Graphical abstract

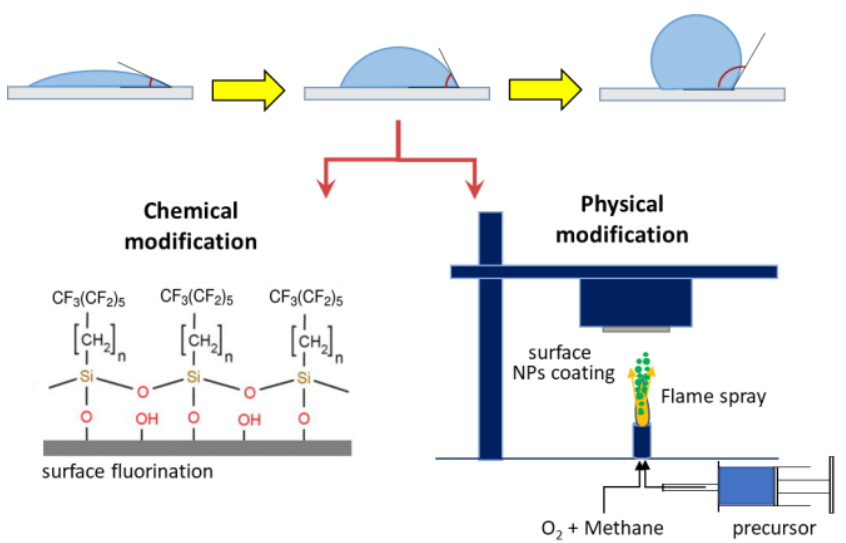

